\title{
Time-dependent analysis of the nuclear and Coulomb dissociation of ${ }^{11} \mathrm{Be}$
}

\author{
Pierre Capel$^{*}$, Gérald Goldstein ${ }^{\dagger}$ and Daniel Baye ${ }^{\dagger}$ \\ ${ }^{*}$ TRIUMF, 4004 Wesbrook Mall, Vancouver, B.C., Canada V6T 2A3 \\ ${ }^{\dagger}$ Physique Quantique, C.P. 165/82 and Physique Nucléaire Théorique et Physique Mathématique, C.P. 229, \\ Université Libre de Bruxelles, B-1050 Brussels, Belgium
}

\begin{abstract}
The breakup of ${ }^{11} \mathrm{Be}$ on carbon and lead targets around $70 \mathrm{MeV} /$ nucleon is investigated within a semiclassical framework. The role of the $\frac{5}{2}^{+}$resonance is analyzed in both cases. It induces a narrow peak in the nuclear-induced breakup cross section, while its effect on Coulomb breakup is small. The nuclear interactions between the projectile and the target is responsible for the transition toward this resonant state. The influence of the parametrization of the ${ }^{10} \mathrm{Be}-\mathrm{n}$ potential that simulates ${ }^{11} \mathrm{Be}$ is also addressed. The breakup calculation is found to be dependent on the potential choice. This leads us to question the reliability of this technique to extract spectroscopic factors.
\end{abstract}

\section{INTRODUCTION}

The ${ }^{11} \mathrm{Be}$ nucleus is one of the best known one-neutron halo nuclei. Its halo structure has thus been the subject of many theoretical and experimental analyzes [1]. In particular, breakup reactions are used as tools to extract its structure properties [2, 3]. Various theoretical models have been developed to interpret the experimental data [4]: perturbation expansion, adiabatic approximation [5], Eikonal model [6], coupled channel with a discretized continuum (CDCC) [7, 8], and numerical resolution of a three-dimensional time-dependent Schrödinger equation [9].

Recently, the breakup of ${ }^{11} \mathrm{Be}$ on both lead and carbon targets has been measured at RIKEN around $70 \mathrm{MeV} / \mathrm{nucleon}$ [3]. In the present talk, we investigate these reactions with a time-dependent technique. This reaction model is based on a semiclassical approximation [9, 10] in which the relative motion of the projectile and the target is approximated by a classical trajectory. Therefore, the projectile is seen as evolving in a time-dependent potential that simulates its interaction with the target. This approximation leads to the resolution of a time-dependent Schrödinger equation. Different techniques have been developed to solve this equation [11, 12, 13, 14, 15, 16]. We use the technique described in Ref. [16].

Up to now, ${ }^{11} \mathrm{Be}$ is described in all reaction models as a two-body system: a halo neutron loosely bound to a structureless ${ }^{10} \mathrm{Be}$ core. The interaction between the neutron and the core is modeled by a simple local potential. This ${ }^{10} \mathrm{Be}-\mathrm{n}$ potential is usually adjusted to reproduce the bound states of ${ }^{11} \mathrm{Be}[11,12,13,14,15]$. It is of course important to analyze the accuracy of that description. In particular, one needs to know what is reproducible using such a simple model, and what is not. In a recent paper, we studied the breakup of ${ }^{11} \mathrm{Be}$ on a ${ }^{12} \mathrm{C}$ target [17]. For that study, we developed a new ${ }^{10} \mathrm{Be}-\mathrm{n}$ potential that reproduces not only the bound states of ${ }^{11} \mathrm{Be}$, but also its first resonant state above the one-neutron threshold. That resonance is found to induce a narrow peak in the breakup cross section. A similar peak is observed in the experimental data. This suggests that the resonance can be fairly well reproduced in the two-body description, and that its presence in reaction models is required to reproduce the experimental data. In this talk, we present the results of this analysis. We also present recent calculations of the Coulomb breakup of ${ }^{11} \mathrm{Be}$. In particular, we discuss the role played by the resonance in that reaction and compare it to its role played in the dissociation on ${ }^{12} \mathrm{C}$.

Besides the capability of this simple two-body description to reproduce physical levels of ${ }^{11} \mathrm{Be}$, the sensitivity of the calculations to the parametrization of the ${ }^{10} \mathrm{Be}-\mathrm{n}$ potential must also be assessed. In particular, the breakup cross section should not be too sensitive to the potential choice if one wishes to reliably extract spectroscopic information from measurements. In this talk, we present the first results of such an analysis. The results of calculations of the Coulomb breakup of ${ }^{11} \mathrm{Be}$ performed with different ${ }^{10} \mathrm{Be}-\mathrm{n}$ potentials are discussed. 


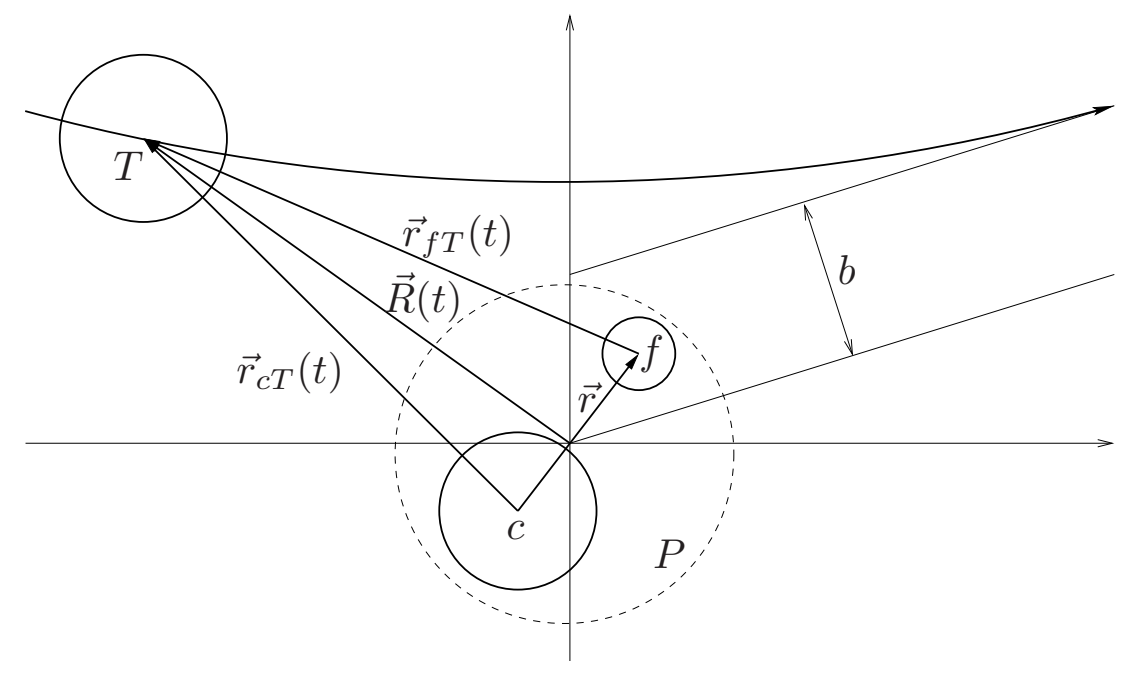

FIGURE 1. Semiclassical scheme of the reaction. In the projectile $(P)$ rest frame, the target $(T)$ follows a classical trajectory described by the time-dependent relative coordinate $\vec{R}$. The projectile is assumed to have a two-body structure: a fragment $f$ loosely bound to a core $c$. Their relative coordinate is $\vec{r}$.

The talk is structured as follows. After a brief description of the time-dependent model and the parametrizations of the potential that describes ${ }^{11} \mathrm{Be}$, we present, in Sec. 3] the results we have obtained in the breakup on ${ }^{12} \mathrm{C}$ [17]. The analysis of the Coulomb breakup of ${ }^{11} \mathrm{Be}$ is discussed in Sec. 4 The final section contains our concluding remarks.

\section{THEORETICAL FRAMEWORK}

\subsection{Time-dependent model}

We consider the breakup of a projectile $P$ by a target $T$. The projectile $P$ is assumed to have a two-body structure: a pointlike and structureless fragment $f$ (of mass $m_{f}$ and charge $Z_{f} e$ ) loosely bound to a structureless core $c$ (of mass $m_{c}$ and charge $Z_{c} e$ ). The target is seen as a structureless particle of mass $m_{T}$ and charge $Z_{T} e$. In the semiclassical approximation [9, 10], the $P-T$ relative motion is treated classically: in the projectile rest frame, the target is assumed to follow a classical trajectory (see Fig. 11. Therefore, the interaction between the projectile and the target is simulated by a time-dependent potential. The internal motion of the projectile, however, is treated quantum mechanically. The wave function $\Psi$ describing this motion is solution of the following time-dependent Schrödinger equation:

$$
\begin{aligned}
i \hbar \frac{\partial}{\partial t} \Psi(\vec{r}, t) & =\left[H_{0}(\vec{r})+V(\vec{r}, t)\right] \Psi(\vec{r}, t) \\
& =\left\{-\frac{\hbar^{2}}{2 \mu} \Delta+V_{c f}(r)+V_{c T}\left[r_{c T}(\vec{r}, t)\right]+V_{f T}\left[r_{f T}(\vec{r}, t)\right]-\frac{\left(Z_{c}+Z_{f}\right) Z_{T} e^{2}}{R(t)}\right\} \Psi(\vec{r}, t),
\end{aligned}
$$

where $\vec{r}$ is the relative coordinate of the fragment to the core, $\vec{R}$ is the time-dependent coordinate that describes the trajectory of the target in the projectile rest frame, and $\vec{r}_{C T}$ and $\vec{r}_{f T}$ are respectively the core-target and fragment-target relative coordinates as illustrated in Fig. 1]

In Eq. (1), the Hamiltonian $H_{0}$ describes the internal structure of the two-body projectile. It is the sum of the kinetic term and the local potential $V_{c f}$, which simulates the interaction between the core and the fragment (see Sec. 2.2. The potentials $V_{c T}$ and $V_{f T}$ model the core-target and fragment-target interactions, respectively. They comprise a Coulomb term and a short-range optical potential, which simulates the nuclear interaction. The latter is usually chosen in the literature.

Eq. (1) is solved with the initial condition that at time $t \rightarrow-\infty$ the projectile is in its ground state. The wave function $\Psi$ at time $t$ is then obtained iteratively using the evolution algorithm described in Ref. [16]. The calculation is 
TABLE 1. Parameters of the ${ }^{10} \mathrm{Be}-\mathrm{n}$ potentials [see Eqs. 3][5]. Note that $R_{0}$ used in (3)-(5) is parametrized as $r_{0} A_{c}^{1 / 3}$.

\begin{tabular}{cccccc}
\hline Potential & $\begin{array}{c}V_{\text {leven }} \\
(\mathrm{MeV})\end{array}$ & $\begin{array}{c}V_{\text {lodd }} \\
(\mathrm{MeV})\end{array}$ & $\begin{array}{c}V_{L S} \\
\left(\mathrm{MeV} \mathrm{fm}^{2}\right)\end{array}$ & $\begin{array}{c}a \\
(\mathrm{fm})\end{array}$ & $\begin{array}{c}r_{0} \\
(\mathrm{fm})\end{array}$ \\
\hline V1 & 62.52 & 39.74 & 21.0 & 0.6 & 1.2 \\
V2 & 66.325 & 38.37 & 12.44 & 0.5 & 1.2 \\
V3 & 58.905 & 40.025 & 27.68 & 0.7 & 1.2 \\
V4 & 71.28 & 49.015 & 29.95 & 0.6 & 1.1 \\
V5 & 55.25 & 32.515 & 12.86 & 0.6 & 1.3 \\
V6 & 59.05 & 59.05 & 0 & 0.62 & 1.236 \\
\hline
\end{tabular}

performed for different trajectories parametrized by the impact parameter $b$. For each trajectory, we deduce the breakup probability by projecting the output wave function $\Psi(\vec{r}, t \rightarrow+\infty)$ onto the positive eigenstates of $H_{0}$ that describe the continuum of the projectile. The breakup cross section is then obtained by summing this probability over all impact parameters.

\section{2. ${ }^{11}$ Be description}

As done in previous works [11, 12, 13, 14, 15, 16, 17], we describe ${ }^{11} \mathrm{Be}$ as a neutron loosely bound to a ${ }^{10} \mathrm{Be}$ core. The ${ }^{10} \mathrm{Be}$ core is assumed to be in its $0^{+}$ground state, and the spectroscopic factor associated to that configuration is set equal to unity. The potential which simulates the ${ }^{10} \mathrm{Be}-\mathrm{n}$ interaction is composed of a central part plus a spin-orbit coupling term

$$
V_{c f}(r)=V_{0}(r)+\vec{L} \cdot \vec{I} V_{L I}(r)
$$

where $\vec{L}$ is the orbital momentum of the ${ }^{10} \mathrm{Be}-\mathrm{n}$ relative motion, and $\vec{I}$ is the spin of the neutron. The central part of $V_{c f}$ has a Woods-Saxon form factor

$$
V_{0}(r)=-V_{l} f\left(r, R_{0}, a\right)
$$

where

$$
f\left(r, R_{0}, a\right)=\left[1+\exp \left(\frac{r-R_{0}}{a}\right)\right]^{-1} .
$$

The spin-orbit coupling term has the usual Thomas form factor

$$
V_{L I}(r)=V_{L S} \frac{1}{r} \frac{d}{d r} f\left(r, R_{0}, a\right) .
$$

The radius of the form factor is parametrized as usual: $R_{0}=r_{0} A_{c}^{1 / 3}$.

The depths of the potential are adjusted to reproduce the energies of the low-lying states of ${ }^{11} \mathrm{Be}$. The well known shell inversion observed between the bound states is reproduced by using a parity-dependent depth of the central part of the potential $V_{l}$. The $\frac{1}{2}^{+}$ground state is modeled by a $1 s 1 / 2$ state, the $\frac{1}{2}^{-}$excited state by a $0 p 1 / 2$ state, and the first $\frac{5}{2}^{+}$resonance is reproduced in the $d 5 / 2$ wave.

In order to study the sensitivity of our calculations to the potential choice, we developed five sets of parameters that reproduce the physical states mentioned above. They are summarized in Table 1 The first potential (V1) has been devised for our recent calculation of the breakup of ${ }^{11} \mathrm{Be}$ on ${ }^{12} \mathrm{C}$ [17] (see also Sec. 3). The next four (V2 to V5) have been obtained by varying either the diffuseness or the radius of the Woods-Saxon form factor. The values were chosen to encompass those used by most other groups [11, 12, 14, 15]. Besides the three physical levels, these potentials all exhibit two unphysical bound states: $0 s 1 / 2$ and $0 p 3 / 2$. These states correspond to the shells occupied by the neutrons in the core and are forbidden by the Pauli principle. Their energies have not been adjusted, and thus vary from one potential to the other. Each potential also displays a $d 3 / 2$ resonance. This resonance does not correspond to 


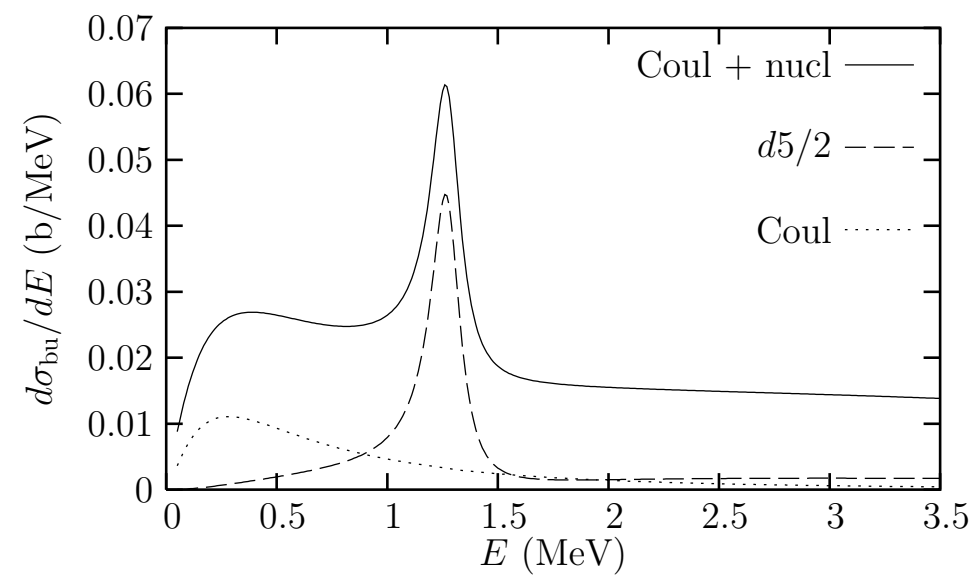

FIGURE 2. Breakup cross section of ${ }^{11} \mathrm{Be}$ on ${ }^{12} \mathrm{C}$ at $67 \mathrm{MeV} /$ nucleon as a function of the ${ }^{10} \mathrm{Be}-\mathrm{n}$ relative energy $E$ after breakup. The full line corresponds to the calculation considering both the nuclear and Coulomb interactions between the projectile and the target. The dashed line displays its $d 5 / 2$ component, responsible for the peak at the resonance energy. The result obtained with a pure Coulomb $P-T$ interaction is displayed as the dotted line.

any known physical state. Therefore it has not been fitted. Its location and width vary with the potential choice. Since it is very broad and located at high energy, we doubt this resonance might play any significant role in our calculations. In Table 1 we also list a sixth potential (V6) developed by Fukuda et al. [3]. It reproduces only the ground state energy and does not contain a spin-orbit coupling term.

\section{BREAKUP OF ${ }^{11} \mathrm{BE} \mathrm{ON}{ }^{12} \mathrm{C}$}

\subsection{Breakup cross section}

Recently, the breakup of ${ }^{11} \mathrm{Be}$ on ${ }^{12} \mathrm{C}$ at $67 \mathrm{MeV} /$ nucleon has been measured at RIKEN [3]. We analyze this reaction within the semiclassical framework described in the previous section. In Fig. 2 the breakup cross section is displayed as a function of the relative energy $E$ between the ${ }^{10} \mathrm{Be}$ core and the neutron after breakup. The full line corresponds to the results obtained considering both nuclear and Coulomb interactions between the projectile and the target. The conditions of the calculations are those described in Ref. [17]. In particular, ${ }^{11} \mathrm{Be}$ is described by the ${ }^{10} \mathrm{Be}-\mathrm{n}$ potential $\mathrm{V} 1$ of Table 1 developed in that previous work. For the ${ }^{10} \mathrm{Be}-{ }^{12} \mathrm{C}$ interaction, we first use the optical potential developed by Al-Khalili, Tostevin and Brook, which has been adjusted to reproduce ${ }^{10} \mathrm{Be}-{ }^{12} \mathrm{C}$ scattering data [18]. The $\mathrm{n}-{ }^{12} \mathrm{C}$ interaction is simulated by the Becchetti and Greenlees parametrization [19]. For comparison, we also display the cross section computed with a pure Coulomb interaction between the projectile and the target (dotted line). This emphasizes the strong dominance of the nuclear interactions in this dissociation reaction.

First, the breakup cross section is significantly enhanced when optical potentials are considered. This is true on the entire energy range, but is particularly striking at high energy. The presence of nuclear $P-T$ interactions leads to a gentle decrease of the cross section with energy, while a pure Coulomb interaction induces a rapid drop of the cross section beyond $0.5 \mathrm{MeV}$.

Second, a narrow peak is observed in the breakup cross section obtained with optical potentials. This peak is due to the $d 5 / 2$ resonance present in our description of ${ }^{11} \mathrm{Be}$. It is indeed located at the same energy and exhibits the same width as that resonance. Moreover, it appears solely in the contribution of the $d 5 / 2$ partial wave to the cross section (dashed line). The absence of peak in the purely Coulomb result indicates that $P-T$ nuclear interactions are necessary to populate that resonant state. 


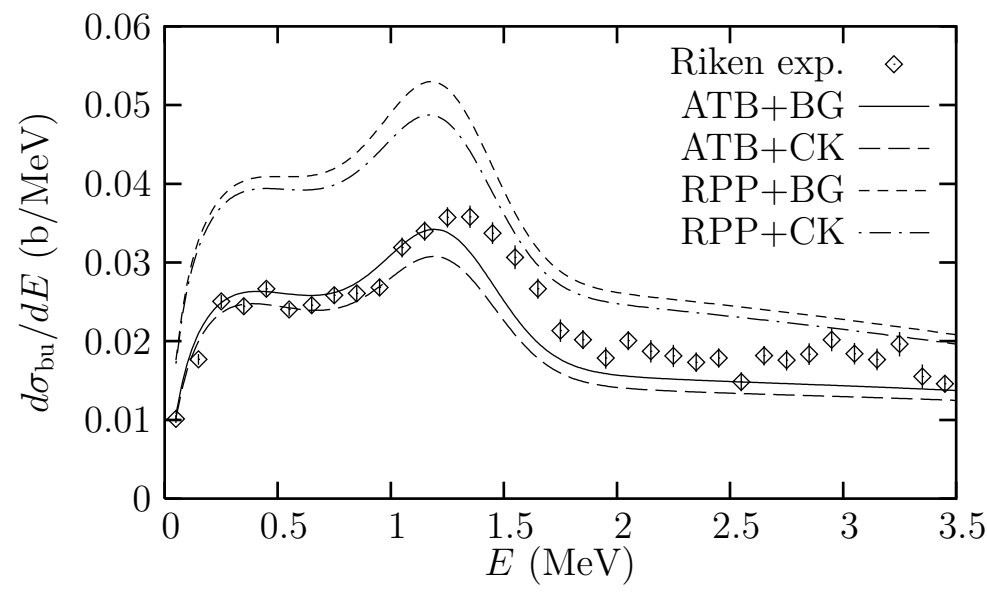

FIGURE 3. Theoretical and experimental breakup cross sections of ${ }^{11} \mathrm{Be}$ on ${ }^{12} \mathrm{C}$ as a function of energy. The four curves correspond to the calculations performed with various combinations of optical potentials simulating the nuclear $P-T$ interactions. The theoretical results have been convoluted with energy resolution. Experimental data are from Ref. [3].

\subsection{Comparison with experiment and analysis of the influence of the optical potentials}

In Fig. 3] we compare the results of our calculation with the breakup cross section measured at RIKEN [3]. The full line (labeled ATB+BG) corresponds to the full line of Fig. 2 convoluted with the experimental energy resolution. The main effect of this convolution is to significantly broaden the resonance peak and slightly shift it toward lower energies.

We observe a very good agreement between theory and experiment. Note that all the parameters have been fixed prior to the calculation; there is no adjustment of our results to the experimental data. At low energy, theory and experiment exhibit the same behavior. In particular, they both display a peak in the vicinity of the $\frac{5}{2}^{+}$resonance. Moreover, these peaks have approximately the same shape (height and width). This confirms that the low-lying resonance in the ${ }^{11} \mathrm{Be}$ spectrum has a significant influence on the nuclear induced breakup.

The discrepancy between theory and experiment observed at larger energies is most likely due to the fact that our

${ }^{11} \mathrm{Be}$ model does not reproduce any known physical state above the $\frac{5}{2}^{+}$one. The experimental ${ }^{11}$ Be spectrum indeed includes two other low-lying resonances, which should have an influence on the breakup as well. The first is located at 2.2 $\mathrm{MeV}$ and is probably responsible for the underestimation of the theory with respect to the experiment. The second is located at $2.9 \mathrm{MeV}$ and may explain the small peak observed in the RIKEN data.

With the aim of analyzing the influence of the parametrization of the nuclear $P-T$ interactions on those results, we perform the same calculation using different sets of optical potentials. Besides the potential of Al-Khalili, Tostevin and Brook [18] (labeled ATB), we choose another potential to simulate the ${ }^{10} \mathrm{Be}-{ }^{12} \mathrm{C}$ nuclear interaction. Following Chatterjee [20], we use a parametrization listed in the Perey and Perey compilation [21] which is a simplified expression of a potential developed by Robson [22] to reproduce the scattering of ${ }^{10} \mathrm{~B}$ on ${ }^{12} \mathrm{C}$ at $18 \mathrm{MeV}$ (labeled RPP). As an alternative to the Becchetti and Greenlees potential (BG) [19] for simulating the $n-{ }^{12} \mathrm{C}$ interaction, we consider the potential developed by Comfort and Karp $(\mathrm{CK})$ to reproduce scattering data of protons impinging on ${ }^{12} \mathrm{C}$ [23].

The breakup cross sections obtained with the four possible combinations of those potentials are displayed in Fig. 3 after convolution with the energy resolution. All curves exhibit the same pattern. In particular, they all display similar peaks near the $\frac{5}{2}^{+}$resonance energy. This result shows that the optical potential choice has but little influence on the shape of that peak. It therefore confirms that the peak reflects the presence of the low-lying resonance in our ${ }^{11} \mathrm{Be}$ model.

The main difference between the four calculations is due to the ${ }^{10} \mathrm{Be}-{ }^{12} \mathrm{C}$ potential. The amplitude of the breakup cross section is indeed multiplied by almost 2 when the ATB potential is substituted by the RPP parametrization. This increase is due to the much smaller imaginary part of RPP. On the other hand, it seems that both $\mathrm{n}-{ }^{12} \mathrm{C}$ interactions are equivalent to describing breakup reactions. The difference between the cross sections obtained with the BG and CK potentials is indeed rather small. 


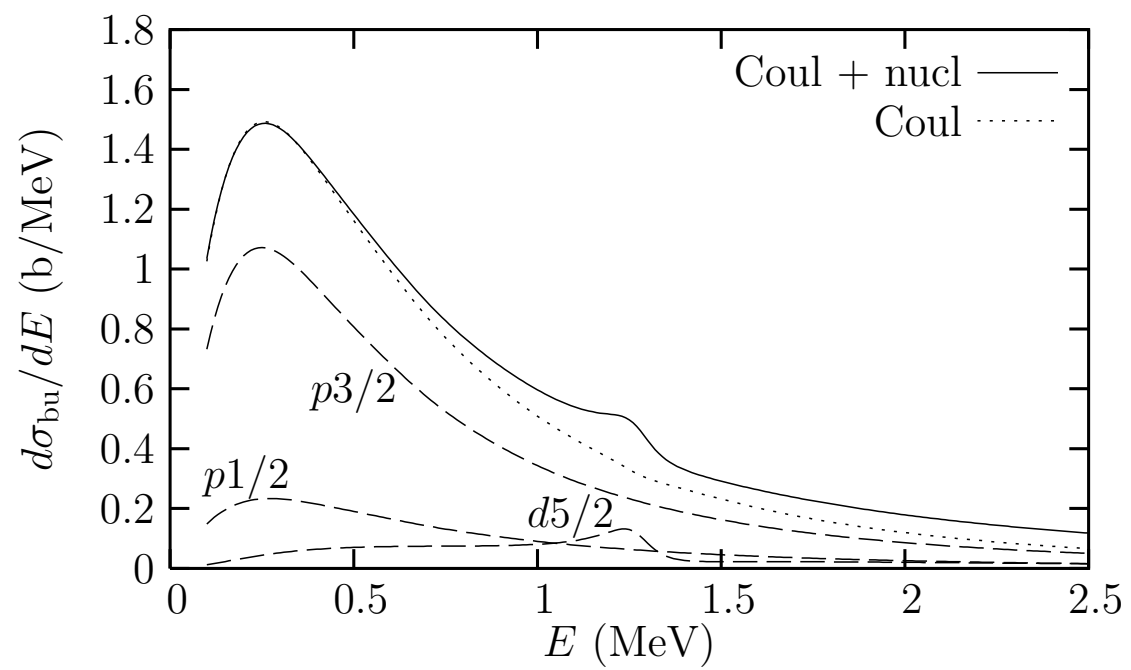

FIGURE 4. Breakup cross section of ${ }^{11} \mathrm{Be}$ on ${ }^{208} \mathrm{~Pb}$ at $69 \mathrm{MeV} /$ nucleon as a function of energy. The full line corresponds to the calculation performed with both Coulomb and nuclear $P-T$ interactions Some major contributions of the partial waves to the cross section are displayed as dashed lines. The dotted line corresponds to the result obtained with a pure Coulomb $P-T$ interaction.

A detailed analysis of the breakup probability as a function of the impact parameter $b$ confirms these results (see Ref. [17]). In particular, it shows that the internal structure of the projectile, like the presence of the $\frac{5}{2}^{+}$resonance, is probed only when the nuclear $P$ - $T$ interactions are taken into account.

These results show that, as expected, the nuclear $P-T$ interactions play a dominant role in the dissociation of halo nuclei on light targets. In particular, these interactions emphasize the presence of low-lying resonances in the projectile spectrum. These resonances must therefore be taken into account in order to reproduce the experimental data. This suggests that nuclear induced breakup can be used as a probe of the continuum spectrum of the projectile.

\section{BREAKUP OF ${ }^{11} \mathrm{BE}$ ON ${ }^{208} \mathrm{~PB}$}

\subsection{Breakup cross section}

We now turn to the Coulomb breakup of ${ }^{11} \mathrm{Be}$ on ${ }^{208} \mathrm{~Pb}$. This reaction has been recently remeasured at RIKEN at $69 \mathrm{MeV} /$ nucleon [3]. Moreover, it is interesting to see how the $\frac{5}{2}^{+}$resonance in the ${ }^{11} \mathrm{Be}$ spectrum will affect the dissociation reaction when it is Coulomb dominated. The breakup cross section is displayed in Fig. 4 4 as a function of the energy $E$. The calculations were performed with the same conditions as in Ref. [16], but for the ${ }^{10} \mathrm{Be}-\mathrm{n}$ potential, which is the same as in Sec. 3.1 (i.e. V1 of Table1).

The full line corresponds to the calculation performed including both Coulomb and nuclear $P-T$ interactions. In this case, the optical potential simulating the nuclear interaction between ${ }^{10} \mathrm{Be}$ and ${ }^{208} \mathrm{~Pb}$ is adapted from an $\alpha$ ${ }^{208} \mathrm{~Pb}$ potential [24] as explained in Ref. [16]. The ${ }^{2}{ }^{208} \mathrm{~Pb}$ potential is chosen to be the Becchetti and Greenlees parametrization [19]. The cross section obtained with a purely Coulomb potential between ${ }^{11} \mathrm{Be}$ and ${ }^{208} \mathrm{~Pb}$ is displayed as a dotted line. In that case, the nuclear interactions are simulated by an impact parameter parameter cutoff.

As expected, the breakup of ${ }^{11} \mathrm{Be}$ on ${ }^{208} \mathrm{~Pb}$ is strongly dominated by the Coulomb interaction. The discrepancy between the cross sections computed with and without the nuclear $P-T$ interactions is indeed small. However, there remain some interesting differences. As in the breakup on ${ }^{12} \mathrm{C}$, but to a much smaller extent, the use of optical potentials leads to an increase of the breakup cross section at high energy. This has already been observed by Typel and Shyam [14]. As explained in Ref. [16], the effect of the nuclear interactions, though small, cannot be fully reproduced by a mere impact parameter cutoff. We also observe that the nuclear $P-T$ interactions induce a small bump in the breakup cross section. As in the previous case, this bump is due to the presence of the $\frac{5}{2}^{+}$state in our description of ${ }^{11} \mathrm{Be}$ : it is located at the resonance energy and is due only to the contribution of the $d 5 / 2$ partial wave (lowest dashed line in 


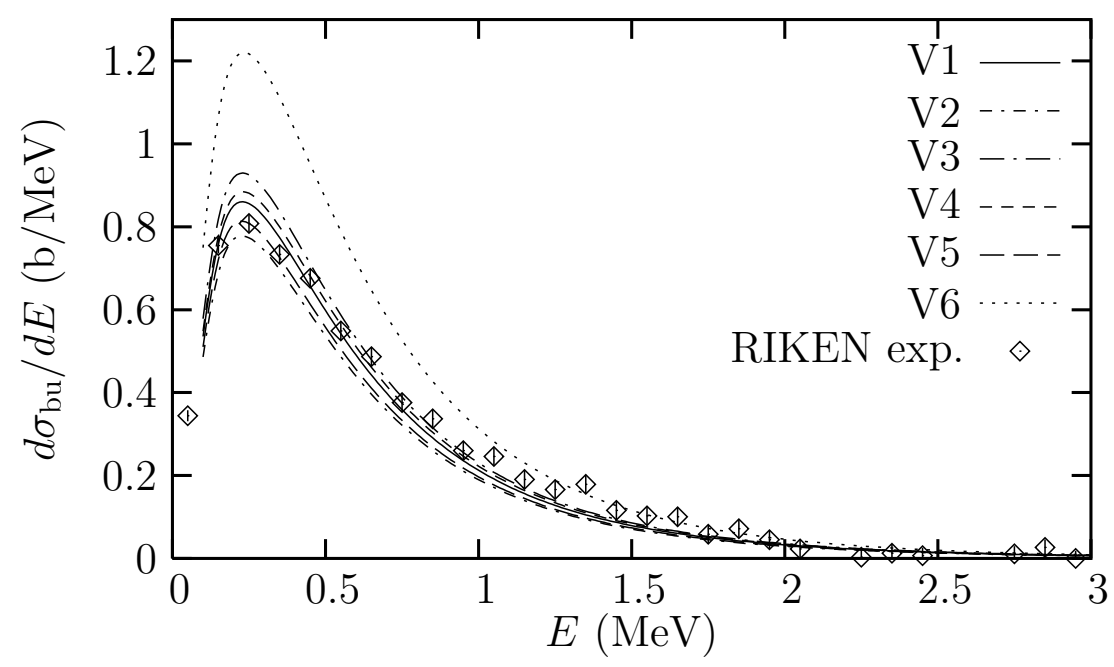

FIGURE 5. Breakup cross sections of ${ }^{11} \mathrm{Be}$ on ${ }^{208} \mathrm{~Pb}$ at $69 \mathrm{MeV} /$ nucleon obtained for $b>30 \mathrm{fm}$. The six curves correspond to calculations performed with the different potentials of Table 1] Experimental data are from Ref. [3].

Fig. (4). It is much smaller than in the previous case (cf. Fig. 2). Although the Coulomb field is very strong in this case, it appears only when optical potentials are used. This effect confirms that only the nuclear interactions can significantly populate the $\frac{5}{2}^{+}$resonant state. Therefore, this low-lying resonance does not affect much the Coulomb dissociation of ${ }^{11} \mathrm{Be}$.

Note that, since the breakup of ${ }^{11} \mathrm{Be}$ on ${ }^{208} \mathrm{~Pb}$ is Coulomb dominated, the calculation of its cross section is much less sensitive to the optical potential choice than in the nuclear induced breakup. A variation of $20 \%$ in the amplitude of the optical potentials leads to only $2 \%$ variation in the breakup cross section.

\subsection{Comparison with experiment and analysis of the sensitivity to the ${ }^{10} \mathrm{Be}-\mathrm{n}$ potentials}

When we compare the results of our calculations with the experimental breakup cross sections measured at RIKEN [3], we find a rather good agreement between theory and experiment. This is illustrated in Fig. [5] where the Coulomb breakup cross section corresponding to $b>30 \mathrm{fm}$ is plotted as a function of the energy. At these impact parameters, the nuclear interactions between projectile and target are completely negligible. This enables us to get rid of the problem of their simulation. The cross section obtained from the calculation presented in the previous section (i.e. using potential V1 of Table 1 is displayed by the full line. This result is indeed very close to the experiment. Note that no parameter has been adjusted to fit the data. In particular, the theoretical cross section has not been scaled by any factor. This suggests that ${ }^{11} \mathrm{Be}$ is well described by a neutron loosely bound to a ${ }^{10} \mathrm{Be}$ core in its $0^{+}$ground state. The spectroscopic factor of that configuration should therefore be close to unity.

With the aim of testing the sensitivity of our results to the ${ }^{10} \mathrm{Be}-\mathrm{n}$ potential, we perform the same calculation with different potentials. These potentials are obtained by varying either the radius or the diffuseness of the Woods-Saxon form factor (see Sec.2.2. They are the potentials V2 to V5 given in Table1 The corresponding breakup cross sections are displayed in Fig. [5 All curves exhibit the same shape. The only difference lies in their amplitude, which varies by about $15 \%$. Surprisingly, these variations are not due to the asymptotic normalization constant of the initial ground state. For example, V4 leads to a larger breakup cross section than V5 (by approximately 9\%), although its ANC (0.82) is smaller than that of V5 (0.87). This puzzling feature is currently under investigation. Up to now, it seems that this difference is due to the scattering properties of the potentials (e.g. scattering length), which differ from one potential to the other. Nevertheless, all these results confirm that the spectroscopic factor of the ${ }^{10} \mathrm{Be}\left(0^{+}\right)$-n configuration should be close to 1 .

For the analysis of their measurements, Fukuda et al. use another ${ }^{10} \mathrm{Be}-\mathrm{n}$ potential (V6 in Table 11] [3]. It is a Woods-Saxon potential whose depth is adjusted only to the ground state energy of ${ }^{11} \mathrm{Be}$. It does not include any 


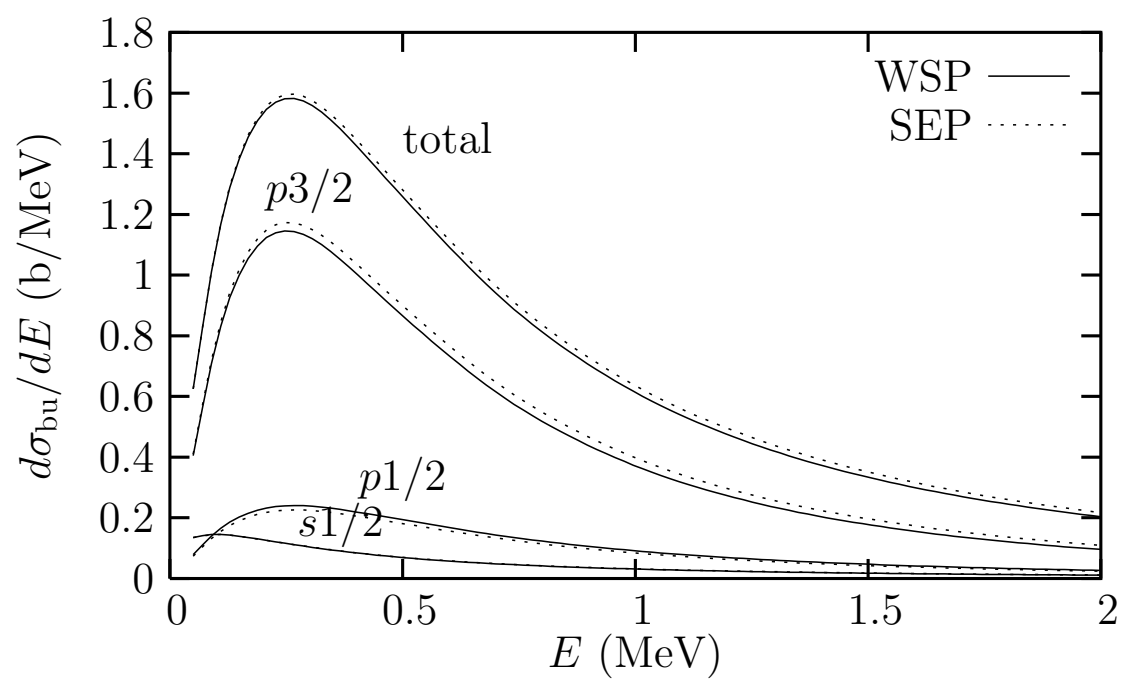

FIGURE 6. Influence of Pauli-forbidden states upon the breakup cross section of ${ }^{11} \mathrm{Be}$ on ${ }^{208} \mathrm{~Pb}$. The full lines correspond to the calculation performed with a Woods-Saxon potential (WSP), while the dotted lines display the results obtained with the supersymmetric equivalent potential (SEP). The latter exhibits the same scattering properties and the same bound spectrum as the former but for the unphysical bound states $0 s 1 / 2$ and $0 p 3 / 2$, which have been removed.

spin-orbit coupling term. From that analysis, they deduce a spectroscopic factor of 0.7 , much lower than what we get from our calculations. In order to understand the discrepancy between our value and theirs, we perform a calculation using potential V6 within our model. The corresponding cross section is displayed as a dotted line in Fig. 5 It lies approximately $30 \%$ above the V1 curve, which explains the lower spectroscopic factor.

The reason for this difference is still to be analyzed. However, the discrepancy obtained within the same reaction model using different $V_{c f}$ supposed to describe the same nucleus is very large. Therefore, we wonder whether this technique is reliable for extracting spectroscopic factors. This result indicates that a strong effort should be made to improve the description of a halo nucleus used in reaction models. At least, the core-fragment potential should be constrained by other experimental data or predictions from precise structure models.

\subsection{Influence of Pauli-forbidden states}

In the preceding section, we saw that the $V_{c f}$ potential used to describe ${ }^{11} \mathrm{Be}$ has a significant influence on the breakup cross section. A thorough analysis of this influence is therefore necessary in order to find a convenient way to constrain the potential choice. A first step in that direction has been done in Ref. [25]. In that previous work, we have studied the influence of the Pauli-forbidden states on the Coulomb breakup of ${ }^{11} \mathrm{Be}$. As explained earlier, ${ }^{11} \mathrm{Be}$ is usually described by deep potentials that exhibit spurious bound states besides the adjusted physical levels. Those unphysical states simulate orbitals occupied by the neutrons of the core. They are thus forbidden to the halo neutron by the Pauli principle. Their presence is usually ignored [11, 12, 13, 14, 15]. However, in reaction models, nothing prevents the transfer of the halo neutron toward one of those spurious states. It is therefore interesting to test the influence of those states upon our calculations.

It is possible to modify a potential in order to remove one of its bound states [26]. The modification consists in a pair of supersymmetric transformations that keeps all the other spectrum properties of the potential unchanged. It means that the supersymmetric partner of the potential exhibits the same scattering properties (i.e. phase shifts) and the same bound spectrum (i.e. energy levels) as the initial potential, except for the bound state that has been removed.

In Ref. [25], we perform two evolution calculations: one with a usual Woods-Saxon potential (WSP), and one with its supersymmetric equivalent potential (SEP) in which both the unphysical $0 s 1 / 2$ and $0 p 3 / 2$ states have been suppressed. The results are shown in Fig. 6 The full lines correspond to the breakup cross section computed with the WSP, and the dotted lines show the result obtained with the SEP. Contributions of the major partial waves are pictured as well. The dependence of the cross section on the potential is very weak. The difference is only $1 \%$ in the 
peak region. Note that the effect differs according to the partial wave. We observe a slight increase $(2.5 \%)$ of the $p 3 / 2$ contribution when the SEP is used. The opposite is obtained for the $p 1 / 2$ component: the SEP cross section is smaller by $5 \%$. The $s 1 / 2$ contribution remains practically unchanged.

This result shows that the Pauli-forbidden states in the projectile spectrum do not play any significant role in the breakup reaction. They may be ignored. The use of deep potentials seems therefore fully justified in such calculations. Moreover, the tiny difference observed between WSP and SEP suggests that the discrepancy between the various ${ }^{10} \mathrm{Be}-\mathrm{n}$ potentials are not directly due to the differences in energies of their unphysical-states.

\section{CONCLUSION}

In this talk, we have presented the results of time-dependent calculations of the nuclear and Coulomb breakup of ${ }^{11} \mathrm{Be}$ [16, 17]. The calculations are performed around $70 \mathrm{MeV} /$ nucleon in order to compare them to recent experimental data [3].

The description of ${ }^{11} \mathrm{Be}$ is improved in comparison to previous works [11, 12, 13, 14, 15, 16]. We developed a new

${ }^{10} \mathrm{Be}$-n potential that reproduces not only the bound states of ${ }^{11} \mathrm{Be}$ but also its low-lying $\frac{5}{2}^{+}$resonance. This resonance has a significant influence on the breakup on ${ }^{12} \mathrm{C}$ [17]: it induces a narrow peak in the breakup cross section. The very good agreement obtained with experiment [3] confirms the validity of the model, and the ability of the timedependent technique to simulate nuclear induced breakup. The spectrum of ${ }^{11} \mathrm{Be}$ includes other low-lying resonances, which should influence the breakup as well. However, the analysis of their actual effect on the cross section requires a better description of the projectile since these resonances cannot be reproduced with such a simple two-body model. In the dissociation on ${ }^{208} \mathrm{~Pb}$, however, the $\frac{5}{2}^{+}$resonance is found to play a rather minor role. It only induces a small bump in the breakup cross section at the resonance energy. In this case also, we obtain a rather good agreement with experimental data [3].

In our reaction model, the nuclear interactions between the projectile and the target are described by optical potentials [16]. In the breakup on ${ }^{12} \mathrm{C}$, they are dominant. In particular, they are found to be responsible for the strong population of the resonant state, which causes the peak in the cross section. The nuclear induced breakup is therefore rather sensitive to the optical potential choice. Using different optical potentials leads to significant variations in the breakup cross section. However, these variations only affect the amplitude of the cross section and not its general pattern. In particular the location and the shape of the resonance peak remain the same for all choices of opticalpotential. The breakup of ${ }^{11} \mathrm{Be}$ on ${ }^{208} \mathrm{~Pb}$ is Coulomb dominated. The nuclear $P$ - $T$ interactions are therefore much less significant, and the cross section is much less dependent on the optical potentials.

The sensitivity of the model onto the ${ }^{11} \mathrm{Be}$ description has also been presented. We performed time-dependent calculations of the Coulomb breakup of ${ }^{11} \mathrm{Be}$ using various ${ }^{10} \mathrm{Be}-\mathrm{n}$ potentials. These potentials lead to cross sections which exhibit the same shape, but differ by up to $30 \%$ in amplitude. This variation is not directly related to the asymptotic normalization constant of the initial ground state. Neither is it to the presence of Pauli-forbidden states in the ${ }^{11} \mathrm{Be}$ spectrum. The role played by these unphysical states in the breakup is indeed negligible [25]. Another effect is thus at play here. Its analysis requires further investigations. Anyway, because of this significant variation in the amplitude of the cross section, using Coulomb breakup as a tool for extracting spectroscopic factors seems questionable.

From this analysis, it seems that we have now reached the limit of the simple two-body description of halo nuclei used in reaction theory. In order to improve our results in the nuclear induced breakup of ${ }^{11} \mathrm{Be}$ we need a more precise model that reproduces the other low-lying resonances. Moreover, the sensitivity of the breakup cross section to the current ${ }^{11} \mathrm{Be}$ model might be too large to extract accurate structure information. Therefore, a reaction model including a more precise description of halo nuclei should be developed in order to improve the theoretical predictions.

\section{ACKNOWLEDGMENTS}

This text presents research results of the Belgian program P5/07 on interuniversity attraction poles initiated by the Belgian-state Federal Services for Scientific, Technical and Cultural Affairs. P.C. acknowledges the support of the Natural Sciences and Engineering Research Council of Canada (NSERC). G.G. acknowledges the support of the FRIA (Belgium). 


\section{REFERENCES}

1. I. Tanihata, J. Phys. G 22, 157 (1996).

2. T. Nakamura, S. Shimoura, T. Kobayashi, T. Teranishi, K. Abe, N. Aoi, Y. Doki, M. Fujimaki, N. Inabe, N. Iwasa, K. Katori, T. Kubo, H. Okuno, T. Suzuki, I. Tanihata, Y. Watanabe, A. Yoshida, and M. Ishihara, Phys. Lett. B 331, 296 (1994).

3. N. Fukuda, T. Nakamura, N. Aoi, N. Imai, M. Ishihara, T. Kobayashi, H. Iwasaki, T. Kubo, A. Mengoni, M. Notani, H. Otsu, H. Sakurai, S. Shimoura, T. Teranishi, Y. X. Watanabe, and K. Yoneda, Phys. Rev. C 70054606 (2004).

4. J. Al-Khalili, and F. M. Nunes, J. Phys. G 29, R89 (2003).

5. R. Johnson, contribution to this workshop (2005).

6. J. Tostevin, contribution to this workshop (2005).

7. I. Thompson, contribution to this workshop (2005).

8. M. Kamimura, contribution to this workshop (2005).

9. D. Baye, contribution to this workshop (2005).

10. K. Alder, and A. Winther, Electromagnetic Excitation, North-Holland, Amsterdam, 1975.

11. T. Kido, K. Yabana, and Y. Suzuki, Phys. Rev. C 50, R1276 (1994).

12. H. Esbensen, G. F. Bertsch, and C. A. Bertulani, Nucl. Phys. A581, 107 (1995).

13. V. S. Melezhik, and D. Baye, Phys. Rev. C 59, 3232 (1999).

14. S. Typel, and R. Shyam, Phys. Rev. C 64, 024605 (2001).

15. M. Fallot, J. A. Scarpaci, D. Lacroix, P. Chomaz, and J. Margueron, Nucl. Phys. A700, 70 (2002).

16. P. Capel, D. Baye, and V. S. Melezhik, Phys. Rev. C 68, 014612 (2003).

17. P. Capel, G. Goldstein, and D. Baye, Phys. Rev. C 70, 064605 (2004).

18. J. S. Al-Khalili, J. A. Tostevin, and J. M. Brooke, Phys. Rev. C 55, R1018 (1997).

19. F. D. Becchetti, Jr., and G. W. Greenlees, Phys. Rev. 182, 1190 (1969).

20. R. Chatterjee, Phys. Rev. C 68, 044604 (2003).

21. C. M. Perey, and F. G. Perey, At. Data Nucl. Data Tables 17, 1 (1976).

22. D. Robson, in Proceedings of the Symposium on Heavy-ion Scattering, edited by R. H. Siemmsen, G. C. Morrison, and J. P. Schiffer, ANL-7837, Argonne National Laboratory, 1971, p. 239.

23. J. R. Comfort, and B. C. Karp, Phys. Rev. C 21, 2162 (1980), ibid. 22, 1809 (E) (1980).

24. B. Bonin, N. Alamanos, B. Berthier, G. Brugea, H. Faraggi, J. C. Lugola, W. Mittigc, L. Papineaub, A. I. Yavind, J. Arvieux, L. Farvacque, M. Buenerd, and W. Bauhoff, Nucl. Phys. A445, 381 (1985).

25. P. Capel, D. Baye, and V. S. Melezhik, Phys. Lett. B 552, 145 (2003).

26. D. Baye, J. Phys. A 20, 5529 (1987). 